\title{
Forced Convection Mass Deposition and Heat Transfer onto a Cylinder Sheathed by Protective Garments
}

\author{
Davide Ambesi and Chris R. Kleijn \\ Dept. of Chemical Engineering, J.M. Burgers Centre for Fluid Mechanics and Delft Process Technology Institute, \\ Delft University of Technology, Julianalaan 136, 2628 BL Delft, The Netherlands
}

Emiel A. den Hartog and Richard H. B. Bouma

TNO Defence, Security and Safety, 2280 AA Rijswijk, The Netherlands

Paul Brasser

Brapa Consultancy, Bleijenburg 144, 2678 BP De Lier, The Netherlands

DOI 10.1002/aic.14246

Published online October 21, 2013 in Wiley Online Library (wileyonlinelibrary.com)

\begin{abstract}
In chemical, biological, radiological, and nuclear protective clothing, a layer of activated carbon material in between two textile layers provides protection against hazardous gases. A cylinder in cross flow, sheathed by such material, is generally used to experimentally test the garment properties. This is, however, complicated and predictive models are needed. We present a computational fluid dynamics model for the initial phase in which the carbon filter material is not yet saturated. The textiles are modeled as chemically inactive porous layers, the carbon filter particles have been resolved explicitly. The model has been validated against experimental data. We demonstrate that (1) computational fluid dynamics simulations can be used for the efficient design and optimization of protective garments, and (2) the addition of a highly porous active carbon layer highly increases the chemical protection capabilities, while having relatively little negative impact on the thermal comfort of protective garments. (C) 2013 American Institute of Chemical Engineers AIChE J, 60: 353-361, 2014 Keywords: chemical, biological, radiological, and nuclear protective garments, cylinder, active carbon filter, heat transfer, mass transfer, computational fluid dynamics, turbulence, time-dependent Reynolds-averaged Navier-Stokes
\end{abstract}

\section{Introduction}

A circular cylinder of approximately $10 \mathrm{~cm}$ in diameter in cross air flow, sheathed by garment material, is a typical model system that is widely used to study the thermal insulation of garments. ${ }^{1-6}$ The system mimics a limb or other body part in clothing. The thermal comfort is primarily determined by the transport of heat and moisture to and from the cylinder. These may be required to be either high or low, depending on the purpose of the garments. The garment material can consist of a single or multiple layers of textile or other materials, and can either be wrapped tightly around the limb, or at some distance with an air layer in between. Typically relevant Reynolds numbers, based on the cylinder diameter and the cross stream velocity, are $10^{3}-10^{5}$. The cross-flow medium being air containing water vapor, relevant Prandtl and Schmidt numbers are of the order of 1 .

Due to its popularity as a model system for garment evaluation, there is a body of literature on heat and moisture transfer to cylinders sheathed by thin porous layers in the above parameter space, as a function of the geometric and material composition of the porous materials, for example,

\footnotetext{
Correspondence concerning this article should be addressed to C. R. Kleijn at C.R.Kleijn@TUDelft.nl.

(C) 2013 American Institute of Chemical Engineers
}

Refs. 7-11. These studies showed the large increase in thermal isolation due to the presence of a thin air layer underneath or in between the porous layers, and the fact that combinations of two layers of moderate air permeability can offer a better isolation than a single layer of low permeability material.

Although the influence of regular garments on heat and mass transfer to body parts is essentially passive, and due to their slowing down and redistributing the air reaching the body part, the role of nowadays chemical, biological, radiological, and nuclear (CBRN) protective clothing is an active one. In such CBRN protective clothing, a thin-usually single-layer of active carbon (either fibers or particles) is embedded in between two textile layers. The purpose of these garments is to prevent CBRN hazardous gases and vapors in the air to reach the skin of the wearer. These gases and vapors are adsorbed by the active carbon. The textile layers serve to slow down and divert the air flow, and as a basis for the attachment of the active carbon filter material. Again, circular cylinders, sheathed at some distance by the protective garment material and exposed to a cross flow of air containing a trace of hazardous gas, are the most commonly studied model systems. ${ }^{3,11}$

In the first hours of their exposure to hazardous gases, that is, before the active carbon becomes saturated, the concentration $C_{\text {out }}$ of hazardous gases in the air that has passed the CBRN protective garment and that reaches the skin is 
typically a low fraction of the concentration $C_{\infty}$ in the incoming air. In this stage, the protection factor (PF), defined as $\mathrm{PF}=C_{\infty} / C_{\text {out }}$, is high and has been found to be almost constant in time. ${ }^{12,13}$ The protection factor is a function of the cross-flow air velocity, the diffusivity of the hazardous gas molecules, the permeability of the textile layers, and the porosity and geometrics of the carbon filter layer. ${ }^{14}$ At later stages, the carbon filter material becomes saturated and PF drops to 1 . Other than the requirement that this should happen as late as possible, this stage is of little relevance as the protective garments should not be worn anymore.

Increasing the packing density of the carbon filter material, and embedding this filter material in low permeability textile layers, reduces the amount of hazardous gas reaching the skin. However, this results in a lower air permeability of the protective clothing, leading to undesired higher heat stresses on the wearer. Such contradictory requirements make the right balance between high protection and acceptable ventilation difficult to achieve. Consequently, design and development of CBRN protective clothing result in a very complex optimization problem. The experimental study and optimization of CBRN protective garments is complicated and time consuming, even when restricted to a simple model configuration such as a single, sheathed cylinder in cross flow. Not only does the PF depend on the characteristics of the external air flow and the hazardous gas, and on the nature of the protective garment material, but also on the location on the cylinder, that is, close to or far from the front stagnation point of the air. This necessitates the development of predictive models.

Brasser proposed analytical models to predict mass deposition onto a cylinder sheathed by passive (i.e., without a layer of active carbon to adsorb hazardous species) ${ }^{15}$ and active $^{16-18}$ garments at high Reynolds numbers. These models contain various assumptions and simplifications, especially regarding the flow distribution around the cylinder and underneath the porous layer.

Whereas in a previous study we reported on the flow and heat transfer around a cylinder covered by a passive textile layer, ${ }^{11}$ the purpose of the present work is twofold: (1) to demonstrate that the addition of a highly porous, high permeability active carbon layer to CBRN protective garments may highly increase its chemical protection capabilities, while having relatively little negative impact on its thermal comfort; (2) to demonstrate that computational fluid dynamics simulations are an accurate and efficient means to describe flow, heat, and mass transfer in active protective garments, and that such simulations can account for relevant complexities which could not be included in earlier analytical models.

To this end, we develop a computational fluid dynamics model for gas deposition onto a cylinder in cross flow, sheathed by a three-layer protective garment consisting of two layers of porous textile and one porous layer of active carbon particles in between. The studied Reynolds numbers (based on free stream velocity and the cylinder diameter) are 12,000 and 34,000 (in order to conform to the experiments by Brasser and van Houwelingen ${ }^{15}$ that we will use to validate our model). Our model provides direct information as to the flow, heat, and concentration distribution around and underneath the active protective garment, without the many simplifying assumptions that were made in earlier studies. ${ }^{15-18}$ The model shows the influence of the free stream Reynolds number, of varying the permeability of the textile layers, and

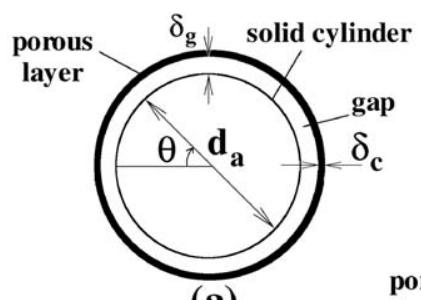

(a)

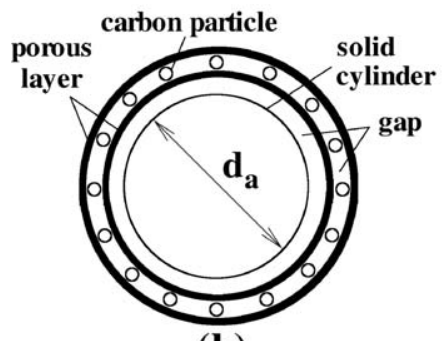

(b)

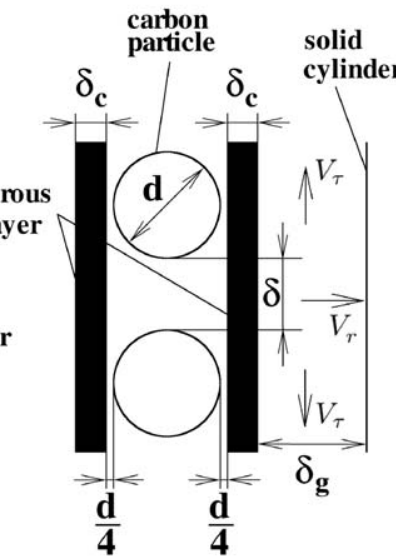

(c)

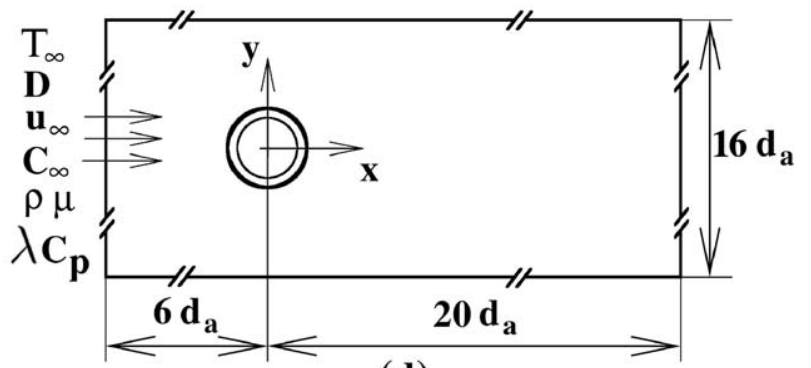

(d)

Figure 1. Tested configurations: (a) solid cylinder sheathed by nonactive protective material; (b) solid cylinder sheathed by active protective material; (c) close-up of the active protective material; (d) computational domain.

of the addition of a carbon layer, on heat transfer and chemical protection. The model can be used to evaluate and optimize the chemical protection and thermal comfort of different designs of protective garments, with respect to the permeabilities of the inner and outer textiles and the porosity and particle size of the carbon filter layer. A model such as presented here may readily be extended and applied to other active carbon filter applications, such as in heating, ventilation, air conditioning (HVAC), cooker hood, and exhaust hood applications.

\section{Numerical Model \\ Problem definition}

Figure 1 shows the studied configurations, viz. a long solid cylinder of diameter $d_{\mathrm{a}}$, with its axis perpendicular to the flow direction, sheathed at some distance $\delta_{\mathrm{g}}$ by either (1) a passive (nonadsorbing) thin porous layer of thickness $\delta_{\mathrm{c}}$ (Figure 1a), or (2) a three-layer protective garment, consisting of two passive porous layers of thickness $\delta_{\mathrm{c}}$ and a single layer of active carbon particles of diameter $d=0.01 d_{\mathrm{a}}$, with a mutual surface-to-surface spacing $\delta$ (Figures $1 \mathrm{~b}, \mathrm{c}$ ). In addition to these configurations, we will also present some results for a bare (that is, nonsheathed) long solid cylinder of diameter $d_{\mathrm{a}}$.

All our simulations are two-dimensional (2-D), and as a consequence the carbon particles are modeled as thin, 
Table 1. Values of the Dimensionless Groups for all Studied Cases

\begin{tabular}{|c|c|c|c|c|c|c|c|c|c|c|}
\hline & Case & $\mathrm{Re}_{\infty}$ & $I_{g}$ & $I_{\mathrm{c}}$ & $\mathrm{Da}_{\text {outer }}$ & $\varepsilon_{\text {filter }}$ & $d / d_{\mathrm{a}}$ & $\mathrm{Da}_{\text {filter }}$ & $\mathrm{Da}_{\text {inner }}$ & $\mathrm{Da}_{\text {total }}$ \\
\hline \multirow[t]{2}{*}{ Bare cylinder } & 1 & 12,000 & - & - & - & - & - & - & - & - \\
\hline & 2 & 34,000 & - & - & - & - & - & - & - & - \\
\hline \multirow[t]{4}{*}{ Passive garment } & 3 & 12,000 & 0.036 & 0.01 & $9.48 \cdot 10^{-6}$ & - & - & - & - & $3.16 \cdot 10^{-6}$ \\
\hline & 4 & 12,000 & 0.036 & 0.01 & $9.48 \cdot 10^{-7}$ & - & - & - & - & $3.16 \cdot 10^{-7}$ \\
\hline & 5 & 34,000 & 0.032 & 0.01 & $2.03 \cdot 10^{-3}$ & - & - & - & - & $6.77 \cdot 10^{-4}$ \\
\hline & 6 & 34,000 & 0.032 & 0.01 & $2.03 \cdot 10^{-4}$ & - & - & - & - & $6.77 \cdot 10^{-5}$ \\
\hline \multirow[t]{4}{*}{ Active garment } & 7 & 12,000 & 0.036 & 0.01 & $9.48 \cdot 10^{-6}$ & 0.6 & 0.01 & $8.00 \cdot 10^{-1}$ & $9.48 \cdot 10^{-5}$ & $2.87 \cdot 10^{-6}$ \\
\hline & 8 & 12,000 & 0.036 & 0.01 & $9.48 \cdot 10^{-6}$ & 0.2 & 0.01 & $4.00 \cdot 10^{-3}$ & $9.48 \cdot 10^{-5}$ & $2.87 \cdot 10^{-6}$ \\
\hline & 9 & 34,000 & 0.032 & 0.01 & $2.03 \cdot 10^{-3}$ & 0.6 & 0.01 & $8.00 \cdot 10^{-1}$ & $2.03 \cdot 10^{-2}$ & $6.13 \cdot 10^{-4}$ \\
\hline & 10 & 34,000 & 0.032 & 0.01 & $2.03 \cdot 10^{-3}$ & 0.2 & 0.01 & $4.00 \cdot 10^{-3}$ & $2.03 \cdot 10^{-2}$ & $4.20 \cdot 10^{-4}$ \\
\hline
\end{tabular}

infinitely long cylindrical fibers with their axes perpendicular to the flow direction. In a previous study, ${ }^{19}$ we have shown that, for a given open frontal area, the mass transfer to such fibers differs little from that to spherical particles. The air gap thickness $\delta_{\mathrm{g}}$ is uniform along the angular direction $\theta$ in all our simulations.

For a prescribed open frontal area fraction $\epsilon$ of the carbon filter, the number of carbon particles $n$ follows from

$$
\epsilon=1-\frac{n d}{2 \pi R}=\frac{n \delta}{2 \pi R}
$$

where $R=d_{\mathrm{a}} / 2+\delta_{\mathrm{g}}+\delta_{\mathrm{c}}+(3 d) / 4$ is the distance between the center of a carbon particle and the center of the solid cylinder. The dimensions of the computational domain are $16 d_{\mathrm{a}}$ in the cross-flow direction $y, 6 d_{\mathrm{a}}$ in the streamwise direction $x$ upstream of the cylinder, and $20 d_{\mathrm{a}}$ in the streamwise direction $x$ downstream of the cylinder, as shown in Figure 1d.

The gas flow is assumed to be isothermal and incompressible. Buoyancy, therefore, does not play a role. The fluid properties of the air-tracer gas mixture (density $\rho$, viscosity $\mu$, thermal conductivity $\lambda$, specific heat capacity $C_{\mathrm{p}}$ and mass diffusivity $D$ of the tracer gas in the air) are assumed to be constant and the gas mixture is assumed to behave as a Newtonian fluid.

We impose a constant inlet gas velocity $u_{\infty}$, as well as a turbulence intensity $I_{\mathrm{t}}=4 \%$ and a turbulence length scale $L_{\mathrm{t}}=d_{\mathrm{a}}$, in the inlet, on the left of the domain. On the walls of the solid cylinder and of the active carbon particles, we impose a no-slip boundary condition for the velocity and standard wall functions ${ }^{20}$ for the turbulence parameters.

At the inlet, we impose a constant tracer gas mass fraction $C_{\infty}=77 \mathrm{mg} / \mathrm{m}^{3}$ (in order to conform to the experiments by Brasser ${ }^{15}$ that we will use to validate our model), and a constant temperature of the flow $T_{\infty}$. As the Peclet number based on $u_{\infty}, D$, and the length of the domain upstream of the cylinder is very large, a purely convective inflow boundary can be used for the tracer species. On the solid walls of the cylinder and the carbon particles, as we study initial breakthrough, the tracer species is assumed to be consumed at an infinite rate, resulting in a zero concentration boundary condition $C_{\mathrm{w}}=0$. The temperature on the surface of the cylinder $T_{\mathrm{w}}$ is maintained constant, whilst the surface of the carbon particles and the porous layers are assumed adiabatic.

The top, lower, and right boundaries of the domain are defined as free outlets, with zero gradients for velocities, concentration, and temperature normal to the boundary. The porous textile layers are modeled as porous media ${ }^{20}$ of prescribed isotropic permeability $K .^{21}$ These act as a momentum sink term in the momentum equations, whereas they are chemically inert.
Meshing of the 2-D domain was performed in Gambit, ${ }^{22}$ using structured meshes in which the solid cylinder and the carbon particles are exclusions from the computational domain. The meshes consisted of approximately 58,000 tetrahedral grid cells for cases in which we studied cylinders sheathed by nonadsorbing material, up to 478,000 grid cells for cases in which we studied cylinders sheathed by a threelayer adsorbing material. Local grid refinements were applied in the region around the carbon particles, in between the two textile layers.

\section{Dimensionless groups}

In addition to the open frontal area fraction $\epsilon$, defined above, and the dimensionless diameter of the particles, which was fixed at $d / d_{\mathrm{a}}=0.01$, six dimensionless groups are defined as follows:

- the free stream Reynolds number $\operatorname{Re}_{\infty}=\rho u_{\infty} d_{\mathrm{a}} / \mu$.

- the Schmidt number $\mathrm{Sc}=\rho /(\mu D)$.

- the Prandtl number $\operatorname{Pr}=\mu C_{\mathrm{p}} / \lambda$.

- the Darcy number $\mathrm{Da}_{i}=K_{i} / \delta_{\mathrm{c}}^{2}$, where $i$ stands for the outer textile layer, the inner textile layer, or the carbon filter layer.

- the porous layer thickness ratio $I_{\mathrm{c}}=\delta_{\mathrm{c}} / d_{\mathrm{a}}$.

- the air gap thickness ratio $I_{\mathrm{g}}=\delta_{\mathrm{g}} / d_{\mathrm{a}}$.

Table 1 summarizes the values used for these dimensionless quantities in all studied cases. Values for $\operatorname{Re}_{\infty}, \mathrm{Sc}, I_{\mathrm{c}}$, $I_{\mathrm{g}}$, and $\mathrm{Da}_{\text {outer }}$ have been chosen in agreement with cases reported by Brasser. ${ }^{15}$ As in protective garments the majority of the pressure drop is caused by the outer textile material, values of $\mathrm{Da}_{\text {inner }}$ were chosen as one order of magnitude larger than those of the outer layer. Also, the carbon filter layer, although not modeled as a porous layer but explicitly resolved in our simulations, can be associated with a permeability $K$, which may be calculated from the theory presented in Ref. 23. These values for $K_{\text {filter }}$ have also been included in Table 1. The total Darcy number of the three-layer garment follows from $\left(d+2 \delta_{\mathrm{c}}\right) \mathrm{Da}_{\text {total }}^{-1}=\delta_{\mathrm{c}} \mathrm{Da}_{\text {outer }}^{-1}+\delta_{\mathrm{c}} \mathrm{Da}_{\text {inner }}^{-1}+$ $d \mathrm{Da}_{\text {filter }}^{-1}$, and is almost entirely determined by the outer textile layer.

Dimensionless output parameters from our simulations include the local Nusselt number $\mathrm{Nu}(\theta)=\phi_{q}^{\prime \prime}(\theta) d_{\mathrm{a}} /$ $\left(\lambda\left(T_{\mathrm{w}}-T_{\infty}\right)\right)$, and the pressure coefficient $C_{\mathrm{p}}(\theta)=(P(\theta)-$ $\left.P_{\infty}\right) /\left(0.5 \rho u_{\infty}^{2}\right)$.

\section{CFD solver and turbulence model}

Computational fluid dynamics models of laminar and turbulent reacting gas flows have been well established and described in literature. ${ }^{24,25}$ To account for the turbulence and the low-frequency oscillations in the flow around the sheathed cylinder, we applied the so-called time-dependent 
Reynolds-averaged Navier-Stokes (T-RANS) approach. This approach solves the time-dependent Navier-Stokes equations with a subgrid turbulence closure model to resolve the large coherent flow structures, in a way that is similar to Large Eddy simulations. ${ }^{26-29}$

Thus, in comparison with traditional RANS, in T-RANS, the subscale model covers only the incoherent, or random, subgrid turbulent motions, whilst the low frequency largescales are fully resolved. This can be done if the characteristic time scale of the coherent motion is sufficiently large compared to the time scale of the turbulent fluctuations. As a subgrid closure model, we use a single-point RANS turbulence closure, ${ }^{26}$ viz. the enormalization group (RNG)-k- $\in$ model. ${ }^{20}$

In this approach, and with the assumptions listed in section Problem Definition, the transient continuity and Navier-Stokes equations read

$$
\begin{gathered}
\frac{\partial \rho}{\partial t}+\frac{\partial}{\partial x_{i}}\left(\rho u_{i}\right)=0 \\
\frac{\partial}{\partial t}\left(\rho u_{i}\right)+\frac{\partial}{\partial x_{j}}\left(\rho u_{i} u_{j}\right)=-\frac{\partial p}{\partial x_{i}}+\frac{\partial}{\partial x_{j}}\left[\mu\left(\frac{\partial u_{i}}{\partial x_{j}}+\frac{\partial u_{j}}{\partial x_{i}}\right)\right]+ \\
\frac{\partial}{\partial x_{j}}\left(-\rho \overline{u_{i}^{\prime} u_{j}^{\prime}}\right)+\alpha S_{i}
\end{gathered}
$$

where $-\rho \overline{u_{i}^{\prime} u_{j}^{\prime}}$ are the components of the Reynolds stress tensor, which represents the effects of subscale turbulence, and $\alpha$ denotes a binary parameter which is equal to 1 in porous regions, and to zero in the fluid region.

$\vec{S}$ is the momentum sink term vector in the porous zone, obtained from the Darcy's law

$$
\vec{S}=-\frac{\mu}{K} \vec{u}
$$

A common approach to close Eq. 3 is to model the Reynolds stress term via the Boussinesq hypothesis ${ }^{30}$

$$
-\rho \overline{u_{i}^{\prime} u_{j}^{\prime}}=\mu_{t}\left(\frac{\partial u_{i}}{\partial x_{j}}+\frac{\partial u_{j}}{\partial x_{i}}\right)-\frac{2}{3}\left(\rho k+\mu_{\mathrm{t}} \frac{\partial u_{i}}{\partial x_{i}}\right) \delta_{i j}
$$

where $\mu_{\mathrm{t}}$ is the turbulent viscosity and $k$ is the turbulent kinetic energy.

The turbulent viscosity can be obtained from a turbulence model. As the standard $k-\epsilon$ model is well known for its weakness in predicting stagnation point flows, ${ }^{31}$ the more accurate RNG- $k-\epsilon$ model $^{20}$ is used instead. Neglecting buoyancy effects, the transport equations for $k$ and $\epsilon$ in this model are

$$
\begin{gathered}
\frac{\partial}{\partial t}(\rho k)+\frac{\partial}{\partial x_{i}}\left(\rho k u_{i}\right)=\frac{\partial}{\partial x_{j}}\left(\alpha_{k} \mu_{\mathrm{eff}} \frac{\partial k}{\partial x_{j}}\right)+G_{\mathrm{k}}-\rho \epsilon \\
\frac{\partial}{\partial t}(\rho \epsilon)+\frac{\partial}{\partial x_{i}}\left(\rho \epsilon u_{i}\right)=\frac{\partial}{\partial x_{j}}\left(\alpha_{\epsilon} \mu_{\mathrm{eff}} \frac{\partial \epsilon}{\partial x_{j}}\right)+C_{1 \epsilon} \frac{\epsilon}{k} G_{\mathrm{k}}-C_{2 \epsilon} \rho \frac{\epsilon^{2}}{k}
\end{gathered}
$$

where $\mu_{\mathrm{eff}}=\mu+\mu_{\mathrm{t}}$, and $G_{\mathrm{k}}$ represents the production of the kinetic energy due to the mean velocity gradients. The model constants are $C_{1 \epsilon}=1.42, C_{2 \epsilon}=1.68, \alpha_{k}=\alpha_{\epsilon}=1.393$. The RNG model provides a differential formula for the effective viscosity that accounts for (local) low Reynolds number effects, ${ }^{20}$ whereas in the high Reynolds regime, $\mu_{\mathrm{t}}$ is calculated as follows

$$
\mu_{\mathrm{t}}=C_{\mu} \rho \frac{k^{2}}{\epsilon}
$$

where $C_{\mu}=0.0845$.
The turbulent mass transport of the tracer species is modeled using the Reynolds analogy to turbulent momentum transfer. In the absence of source terms due to chemical reactions in the gas phase, the trace gas mass transport equation is given by

$$
\frac{\partial}{\partial t}\left(\rho C_{i}\right)+\frac{\partial}{\partial x_{i}}\left(\rho u_{i} C_{i}\right)=\frac{\partial}{\partial x_{i}}\left[\left(\rho D+\frac{\mu_{t}}{\mathrm{Sc}_{\mathrm{t}}}\right) \frac{\partial C_{i}}{\partial x_{i}}\right]
$$

where $C_{i}$ is the mass fraction of the tracer species and $\mathrm{Sc}_{\mathrm{t}}$ is the turbulent Schmidt number. ${ }^{20}$ Because of the low concentrations of hazardous gas, the laminar diffusion, which is dominant underneath the garments can be accurately modeled using Fick's law. ${ }^{32}$

A second-order QUICK scheme ${ }^{33}$ was used to discretize the equations in space, whereas a second-order implicit scheme was used to discretize the equations in time. The time step $\Delta t$ was set equal to $1 / 20$ of the time scale $\tau$ of the vortex shedding behind the cylinder. ${ }^{34}$ For the studied range of Reynolds numbers, the Strouhal number $\mathrm{St}=u_{\infty} \tau / d_{\mathrm{o}}$ is approximately $0.2,{ }^{34}$ or $\tau \approx 0.2 d_{\mathrm{o}} / u_{\infty}$, where $d_{\mathrm{o}}$ is the outer diameter of the cylinder plus porous layers. The pressure field was obtained by using the SIMPLE algorithm. ${ }^{20}$ Convergence limits for the sum of the normalized absolute residuals for all the equations were set to $10^{-6}$ at each time step.

\section{Results and Discussion \\ Model validation for an unsheathed cylinder}

Before presenting our results for mass deposition onto a cylinder in cross flow sheathed by a thin porous layer, we discuss the ability of our simulation approach to accurately predict the flow around, and heat and mass transfer to, a nonsheathed cylinder in cross flow at relevant values of $\mathrm{Re}_{\infty}$. In an earlier study, ${ }^{29}$ we showed that our T-RANS simulations with an RNG-k- $\epsilon$ turbulence model are capable of predicting drag coefficients and mean streamwise velocities at the centerline behind the cylinder in good agreement with experimental data. Here, we add results for the local Nusselt number $\mathrm{Nu}(\theta)$ and the dimensionless pressure coefficient $C_{\mathrm{p}}(\theta)$ on the cylinder surface, compared to experimental data.

Figure 2 shows that the computed values and the experimental findings are in very good agreement. The fact that the experimentally observed local minimum in $\mathrm{Nu}$ at the stagnation point $\theta=0^{\circ}$ is not found in the simulations can be linked to the well known tendency of $k-\epsilon$ turbulence models to over-predict mass and heat transfer coefficients in stagnation points. ${ }^{31}$ The Strouhal number St of the vortex shedding in our simulations was found to be $\mathrm{St}=0.2143$ and $\mathrm{St}=0.2104$ for $\mathrm{Re}_{\infty}=12,000$ and 34,000 , in good agreement with the values $\mathrm{St}=0.2136$ and $\mathrm{St}=0.2097$, respectively, predicted by the empirical correlation of Norberg. ${ }^{34}$ All these findings were checked to be highly insensitive to doubling the amount of grid cells in both directions, varying the inlet turbulence intensity from 2 up to $8 \%$, as well as varying the inlet turbulent length scale from 0.5 to $2 d_{\mathrm{a}}$.

\section{Model validation for a cylinder sheathed by a passive porous layer}

As a further validation of our simulation approach, we present results from our model for a cylinder sheathed by a single, passive (i.e., nonadsorbing), textile layer. We study the mass deposition $M_{\mathrm{d}}$, during an exposure time $\Delta t=45$ 

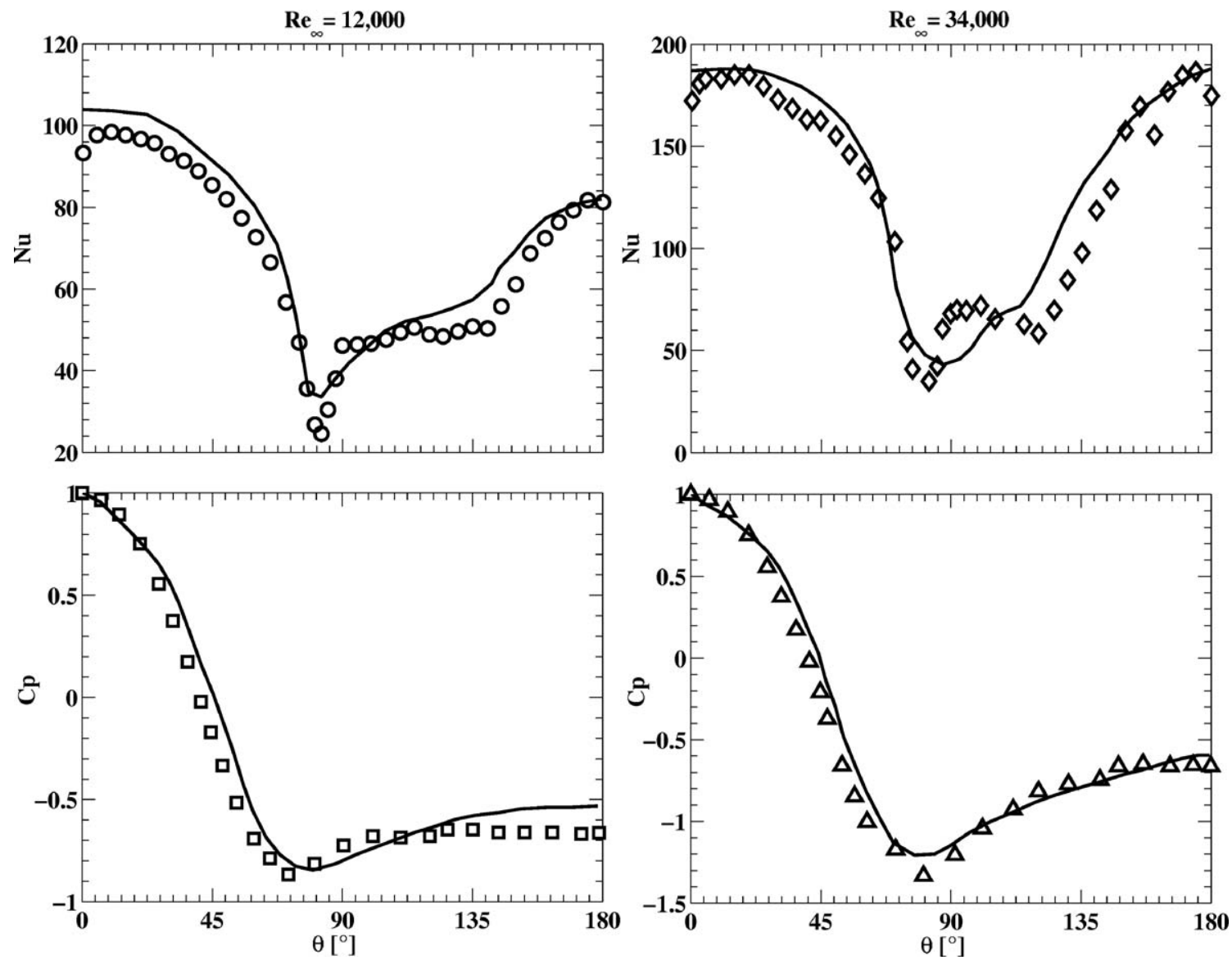

Figure 2. Angular distribution of the Nusselt number $\mathrm{Nu}$ and the pressure coefficient $C_{\mathrm{p}}$ for a bare cylinder in cross flow $(\operatorname{Pr}=0.7)$ : experimental data from Labraga and Berkah for $\operatorname{Re}_{\infty}=8,500^{35}(\bigcirc)$; experimental data from Petrie and Simpson for $\operatorname{Re}_{\infty}=10,100^{36}(\square)$; experimental data from Scholten and Murray for $\mathbf{R e}_{\infty}=35,950^{37}$ ( $\left.\square\right)$; experimental data from Petrie and Simpson for $\mathbf{R e}_{\infty}=34,200^{36}(\Delta)$; present simulations for $\operatorname{Re}_{\infty}=12,000$ and $\operatorname{Re}_{\infty}=34,000$ (solid-line, left and right column, respectively).

min to an airflow with a tracer gas concentration $C_{\infty}=77$ $\mathrm{mg} / \mathrm{m}^{3}$, onto the surface of the sheathed cylinder, for the conditions of Cases 3 and 5 in Table 1, and compare our results to those reported experimentally by Brasser. ${ }^{15}$

Figure 3 shows the distributions of the viscosity ratio $\mu_{\mathrm{eff}} /$ $\mu$, and the dimensionless temperature $\Gamma=\left(T-T_{\mathrm{w}}\right) /\left(T_{\infty}-T_{\mathrm{w}}\right)$, in the vicinity of the stagnation point, both outside and beneath the porous garment layer. It can clearly be seen that the flow, which is highly turbulent outside the garment, becomes laminar $\left(\mu_{\mathrm{eff}} / \mu \simeq 1\right)$ underneath the garment layer. This is in agreement with our earlier results from direct numerical simulation (DNS) simulations. ${ }^{38} \mathrm{~A}$ thin thermal boundary layer is formed underneath the garment, the thickness of which is very small compared to the cylinder diameter and is thinnest in the stagnation point. This results in Nusselt values which are much larger than one everywhere along the cylinder surface, and which have a maximum in the stagnation point.

Figure 4 shows that the computed values of the mass deposition onto the cylinder surface $M_{\mathrm{d}}$ agree well with those found in the experiments by Brasser et al. ${ }^{15}$ The accuracy of our approach is also shown in Figure 5, where the experimental and the computed deposition velocities $V_{\mathrm{d}}=M_{\mathrm{d}} /\left(C_{1 / 2} \Delta t\right)$ (with $C_{1 / 2}$ the tracer species concentration underneath the garment, at $\left.r=d_{\mathrm{a}} / 2+\delta_{\mathrm{g}} / 2\right)$ are compared. As was done by Brasser, ${ }^{15}$ values of $V_{\mathrm{d}}$ are plotted against the radial velocity $V_{\mathrm{r}}$ through the porous material for $\theta=0^{\circ}$, and against the tangential velocity $V_{\tau}$ underneath the protective material for at $\theta=45^{\circ}$, both for for $\operatorname{Re}_{\infty}=12,000$ and 34,000 . The computed results confirm that $V_{\mathrm{d}}$ can be well described as a function of the square root of $V_{\mathrm{r}}$ or $V_{\tau}$, as suggested by Brasser. ${ }^{15}$

\section{Mass deposition and heat transfer for a cylinder sheathed by a three-layer adsorbing porous material}

In this section, we address the influence of adding an active porous carbon layer and a second-textile layer to the garment, thus creating a three-layer protective garment around the cylinder. The focus will be on the increased chemical protection and the decreased heat transfer, as compared to a single-passive textile layer only.

Figures 6 and 7 show the values of the predicted mass deposition $M_{\mathrm{d}}$ and Nusselt number $\mathrm{Nu}$, as a function of the angular position $\theta$, for cylinders covered by single, passive textile layers, and by three-layer active garments, for conditions as given in Table 1 . Cases 3 and 5, for cylinders covered by a passive single-textile layer, are identical to those shown in Figure 4. The experimental data from Brasser et al. ${ }^{15}$ for Cases 3 and 5 have also been included again.

In Case 4, the hydraulic permeability of the single-passive textile layer is reduced by a factor 10 compared to Case 3 . 

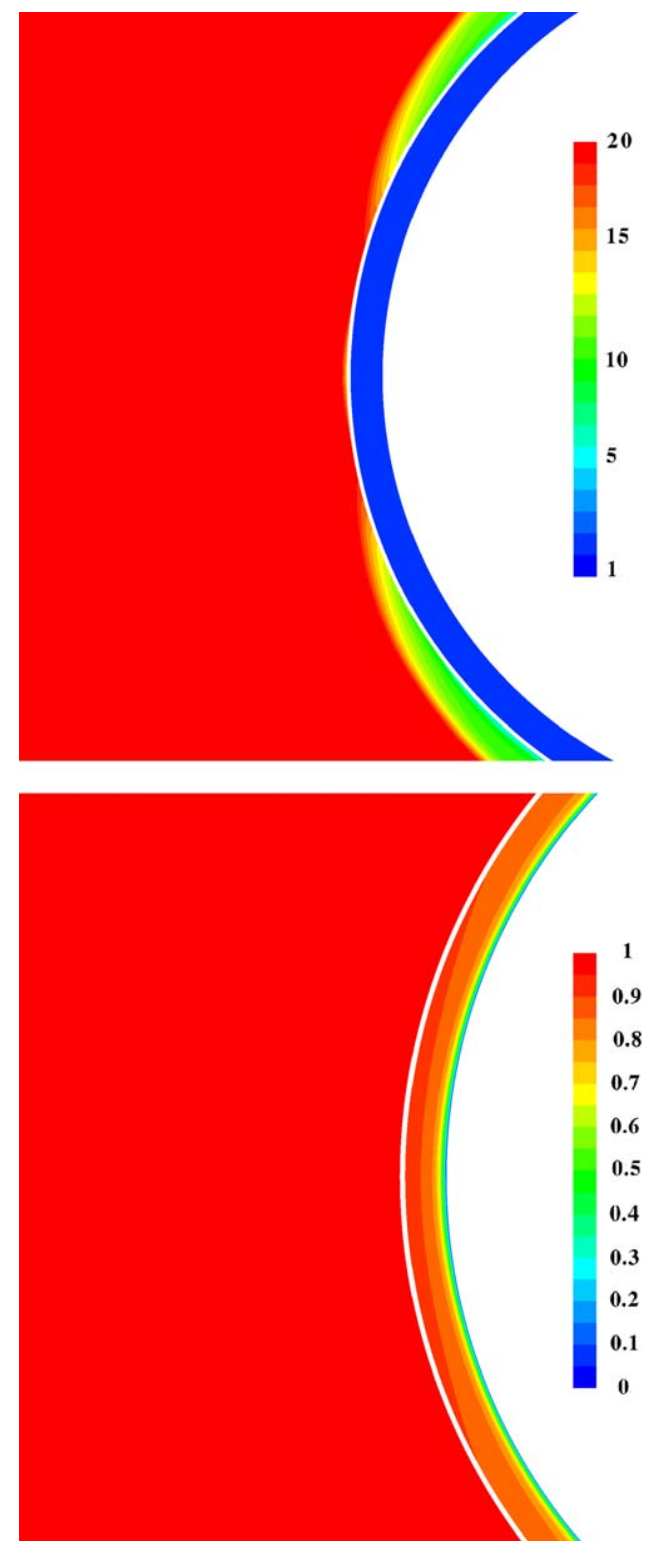

Figure 3. Stagnation point close-up for a cylinder sheathed by a nonadsorbing single layer for the conditions of Case 5.

Snapshot at $t=20 \mathrm{~T}$; contours of the ratio between effective viscosity $\mu_{\text {eff }}$ and laminar viscosity $\mu_{\mathrm{lam}}$ (top); contours of the dimensionless temperature (bottom). White enclosures represent the porous textile layer. [Color figure can be viewed in the online issue, which is available at wileyonlinelibrary.com.]

This leads approximately to a factor 10 decrease in the velocities through and underneath the garment. Since, as shown in Figure 5, the mass transfer rate (and consequently also the heat transfer rate) to the cylinder scales with the square root of these velocities, both $M_{\mathrm{d}}$ and $\mathrm{Nu}$ are reduced by approximately a factor 3 for all $\theta$, compared to Case 3 . The same is observed when comparing Cases 5 and 6 in Figure 7. Clearly, applying less permeable textile material, without the addition of an active filter layer, leads to a (limited) improvement in the chemical protection, but at the same time leads to an equally large decrease in the thermal comfort.

In contrast to this, by comparing Cases 3 and 7 in Figure 6, it can be seen that the addition of a high porosity

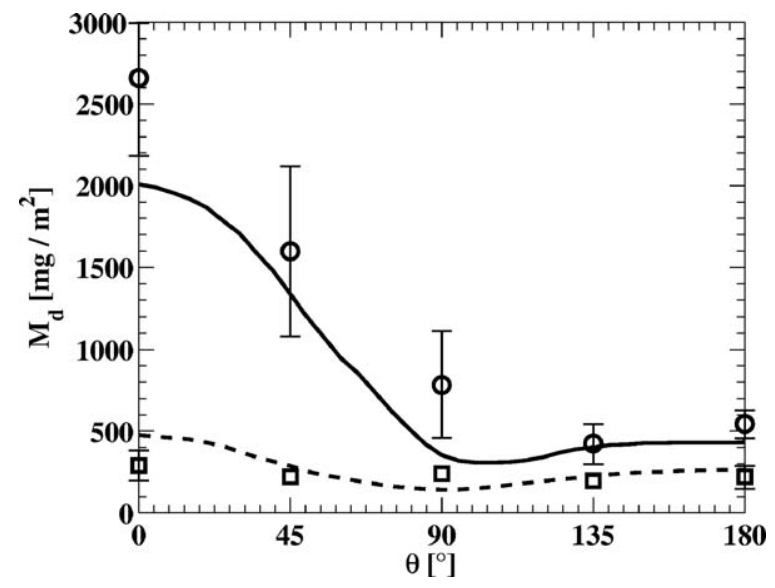

Figure 4. Angular dependence of the mass deposition onto a solid cylinder sheathed by a passive porous layer, after $\Delta t=\mathbf{4 5} \mathrm{min}$ of exposure to an air flow with a tracer gas concentration $C_{\infty}=77 \mathrm{mg} / \mathrm{m}^{3}$ : experimental data $(\bigcirc)$ from Brasser et al., ${ }^{15}$ and present simulation results (solid line) for conditions as in Case 3 of Table 1; experimental data $\square$ ) from Brasser et al., ${ }^{15}$ and present simulation results (dashed line) for conditions as in Case 5 of Table 1.

$(\epsilon=0.6)$, high permeability active carbon layer (in combination with a high permeability inner textile layer) leads to a three orders of magnitude decrease in the mass deposition, whereas $\mathrm{Nu}$ in the stagnation point is decreased by only some $25 \%$. The latter is not primarily due to the slight decrease in permeability, as compared to the single-textile layer, but mostly due the fact that part of the air that has penetrated the outer textile layer stays in between the outer

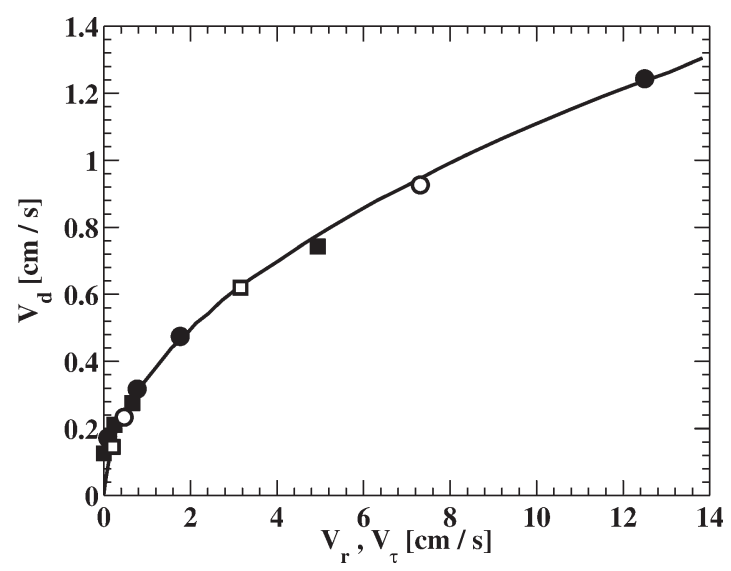

Figure 5. Deposition velocity $V_{d}=M_{d} /\left(C_{1 / 2} \Delta t\right)$ as function of the air speed $\left(V_{r}\right)$ through the garment at $\theta=0^{\circ}$, and the air speed $\left(V_{\tau}\right)$ underneath the garment at $\theta=45^{\circ}$ : experimental data $(\bullet)$ from Brasser et al., ${ }^{15}$ and present simulation results $(O)$ at $\theta=0^{\circ}$ for conditions as in Cases 3 and 5 of Table 1; experimental data (घ) from Brasser et al., ${ }^{15}$ and present simulation results $\square$ ) at $\theta=45^{\circ}$ for conditions as in Cases 3 and 5 of Table 1; curve fit $V_{d} \propto$ $\left(\boldsymbol{V}_{\mathbf{r}}, \boldsymbol{V}_{\tau}\right)^{0.5}$ (solid-line). 


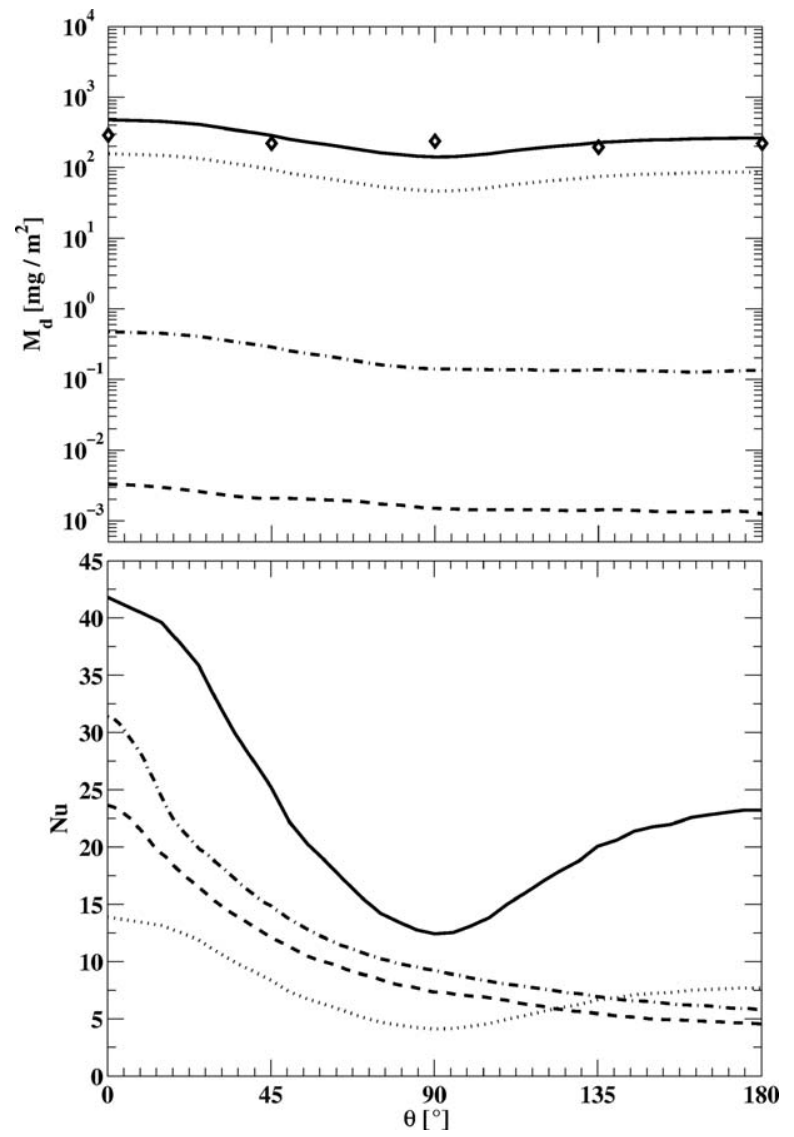

Figure 6. Mass deposition (upper graph) and Nusselt number (lower graph) to bare and sheathed cylinders at $\operatorname{Re}_{\infty}=12,000$ and other conditions as in Table 1.

Case 3 (solid line); Case 4 (dotted line); Case 7 (dasheddotted line); Case 8 (dashed line); experimental data from Brasser et al. ${ }^{15}$ for Case $3(\square)$.

and inner textile layer and flows tangentially between these layers, rather than impinging to the cylinder in the stagnation point. This can also be observed from the modified distribution of $\mathrm{Nu}(\theta)$ along the cylinder surface, as compared to that for Case 3. For the high-permeability, high $R e_{\infty}$ Case 5 shown in Figure 7, the addition of a very open $(\epsilon=0.6)$ single-active carbon layer attached to a second, very highpermeability textile layer, as in Case 9, leads to a sevenfold decrease in $M_{\mathrm{d}}$, whereas $\mathrm{Nu}$ in the stagnation point is decreased by only some $20 \%$.

Both for the low permeability, low $\operatorname{Re}_{\infty}$ case in Figure 6 and for the high permeability, high $\operatorname{Re}_{\infty}$ case in Figure 7, the addition of more dense $(\epsilon=0.2)$ active carbon layer even leads to significant further decreases in $M_{\mathrm{d}}$. Comparing Cases 3 and 8 , we see a five orders of magnitude improvement in chemical protection at the expense of a less than $50 \%$ decrease in the stagnation point Nu. Similarly, comparing Cases 5 and 10, we see a 20-fold improvement in chemical protection at the expense of a $25 \%$ decrease in the stagnation point $\mathrm{Nu}$. These examples show how the inclusion of a single layer of active carbon particles or fibers in protective clothing may lead to highly increased chemical protection, at the expense of a relatively limited decrease in thermal comfort.

\section{Conclusions}

In this work, we demonstrated the applicability of computational fluid dynamics simulations to model air flow, heat transfer, and mass deposition onto the surface of a cylinder in turbulent cross flow, sheathed by CBRN protective garment material. The main conclusions from our work are:

- Unlike earlier analytical models, that over-simplify the flow around and underneath the garment material, computational models may be used to accurately and efficiently optimize three-layer active CBRN protective clothing material, for example, by varying the permeabilities of the inner and outer textiles and by varying the porosity and particle size of the carbon filter layer. Computational simulations give room for informed and quantifiable decisions on designing clothing with an optimal balance between protection and thermal strain for various conditions.

- Because of the turbulent and periodic nature of the flow around the cylinder, and the laminarization of the flow underneath the garment, the simulations should be based on an advanced form of turbulence modeling. Our study showed that the T-RANS approach is very well suited for this purpose. The simulations proved to

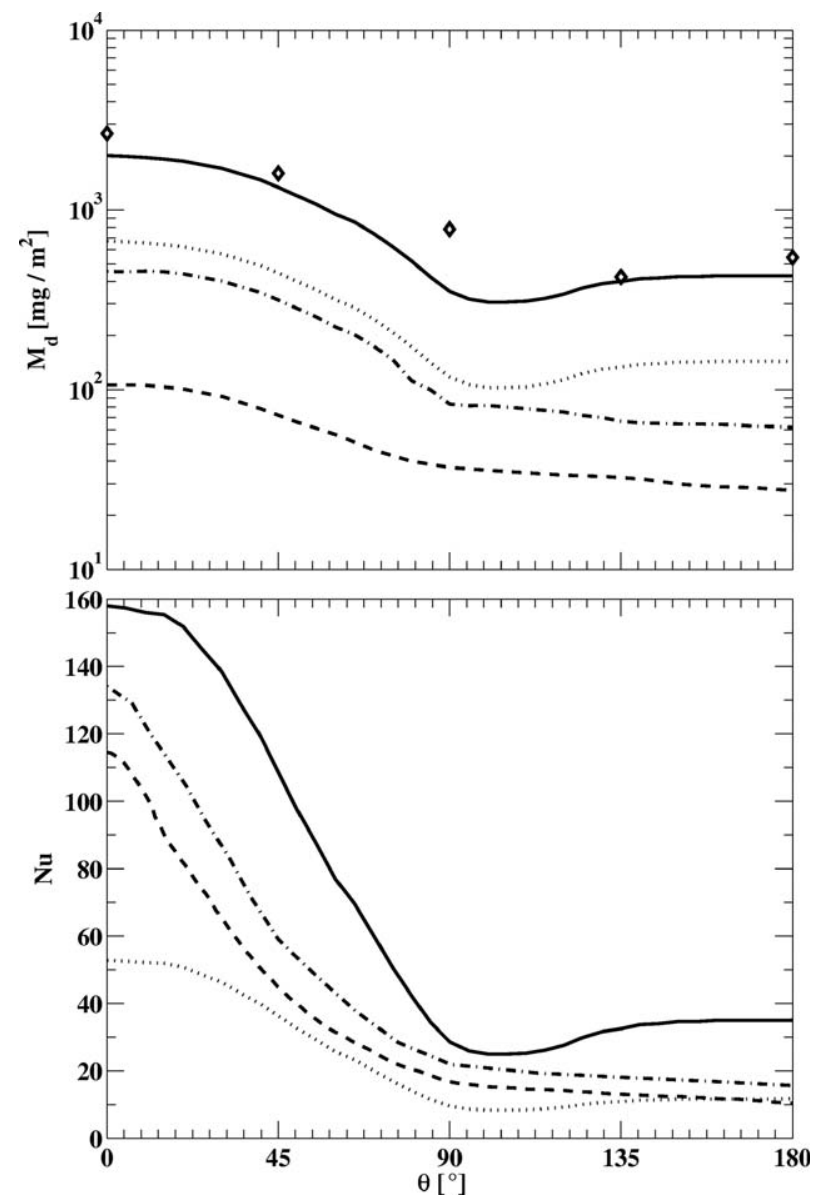

Figure 7. Mass deposition (upper graph) and Nusselt number (lower graph) to bare and sheathed cylinders at $\operatorname{Re}_{\infty}=34,000$ and other conditions as in Table 1.

Case 5 (solid line); Case 6 (dotted line); Case 9 (dasheddotted line); Case 10 (dashed line); experimental data from Brasser et al. ${ }^{15}$ for Case 5 ( $\square$ ). 
render results that were in good agreement with published experimental data for bare cylinders, and for cylinders sheathed by a single passive textile layer.

- For realistic conditions as used in testing protective garments, the introduction of a high porosity single layer of activated carbon particles reduces the mass deposition of tracer gas onto the cylinder surface by several orders of magnitude, whereas heat transfer is decreased by tens of percents only.

- A model such as presented here may easily be extended and applied to other active carbon filter applications, such as in HVAC, cooker hood, and exhaust hood applications.

\section{Notation}

$C_{\text {out }}=$ tracer gas concentration in filtered air, $\mathrm{kg} / \mathrm{m}^{3}$

$C_{\mathrm{p}}=$ specific heat, $\mathrm{J} /(\mathrm{kgK})$

$C_{\mathrm{w}}=$ tracer gas concentration at the walls, $\mathrm{kg} / \mathrm{m}^{3}$

$C_{\infty}=$ tracer gas concentration in the free stream, $\mathrm{kg} / \mathrm{m}^{3}$

$d=$ diameter of active carbon particles, $\mathrm{m}$

$D=$ tracer gas mass diffusivity, $\mathrm{m}^{2} / \mathrm{s}$

$d_{\mathrm{a}}=$ diameter of the solid cylinder, $\mathrm{m}$

$d_{\mathrm{o}}=$ outer diameter of the sheathed cylinder, $\mathrm{m}$

$I_{\mathrm{t}}=$ turbulence intensity

$k=$ turbulent kinetic energy, $\mathrm{m}^{2} / \mathrm{s}^{2}$

$K=$ isotropic permeability, $\mathrm{m}^{2}$

$L_{\mathrm{t}}=$ turbulence length scale, $\mathrm{m}$

$M_{\mathrm{d}}=$ deposited mass, $\mathrm{kg} / \mathrm{m}^{2}$

$n=$ number of carbon particles

$P(\theta)=$ local pressure, $\mathrm{Pa}$

$P_{\infty}=$ free stream pressure, $\mathrm{Pa}$

$\mathrm{PF}=$ protection factor

$\vec{S}=$ momentum sink term vector, $\mathrm{Pa} / \mathrm{m}$

$T_{\mathrm{w}}=$ cylinder wall temperature, $\mathrm{K}$

$T_{\infty}=$ free stream fluid temperature, $\mathrm{K}$

$u_{\infty}=$ free stream fluid velocity, $\mathrm{m} / \mathrm{s}$

$V_{\mathrm{d}}=$ deposition velocity, $\mathrm{m} / \mathrm{s}$

$V_{\mathrm{r}}=$ radial velocity through the porous layer, $\mathrm{m} / \mathrm{s}$

$V_{\tau}=$ tangential velocity underneath the protective material, $\mathrm{m} / \mathrm{s}$

$\vec{u}=$ velocity vector, $\mathrm{m} / \mathrm{s}$

$x=$ streamwise direction

$y=$ cross-flow direction

$\delta=$ distance between carbon particles, $\mathrm{m}$

$\delta_{\mathrm{c}}=$ thickness of the passive porous layer, $\mathrm{m}$

$\delta_{\mathrm{g}}=$ distance between the sheathed cylinder and the first passive porous layer, $\mathrm{m}$

$\Delta t=$ simulation time step, $\mathrm{s}$

$\in=$ open frontal area fraction of the carbon filter

$\Gamma=$ dimensionless temperature

$\phi_{q}^{\prime \prime}=$ heat flux, $\mathrm{W} / \mathrm{m}^{2}$

$\lambda=$ thermal conductivity, $\mathrm{W} /(\mathrm{mK})$

$\mu=$ dynamic viscosity, $\mathrm{kg} /(\mathrm{ms})$

$\mu_{\mathrm{t}}=$ turbulent viscosity, $\mathrm{kg} /(\mathrm{ms})$

$v=$ kinematic viscosity, $\mathrm{m}^{2} / \mathrm{s}$

$\rho=$ fluid density, $\mathrm{kg} / \mathrm{m}^{3}$

$\tau=$ time scale of vortex shedding, $\mathrm{s}$

$\theta=$ angular direction

$C_{\mathrm{p}}(\theta)=$ pressure coefficient $\left(P(\theta)-P_{\infty}\right) /\left(0.5 \rho u_{\infty}^{2}\right)$

$\mathrm{Da}=$ Darcy number $K / \delta_{\mathrm{c}}^{2}$

$I_{\mathrm{c}}=$ porous layer thickness ratio $\delta_{\mathrm{c}} / d_{\mathrm{a}}$

$I_{\mathrm{g}}=$ air gap thickness ratio $\delta_{\mathrm{g}} / d_{\mathrm{a}}$

$\mathrm{Nu}=$ local Nusselt number $\phi_{q}^{\prime \prime \prime}(\theta) d_{\mathrm{a}} /\left(\lambda\left(T_{\mathrm{w}}-T_{\infty}\right)\right)$

$\operatorname{Pr}=$ Prandtl number $\mu /\left(C_{\mathrm{p}} \lambda\right)$

$\operatorname{Re}_{\infty}=$ Reynolds number $\rho u_{\infty} d_{\mathrm{a}} / \mu$

$\mathrm{Sc}=$ Schmidt Number $\rho /(\mu D)$

$\mathrm{St}=$ Strouhal number $u_{\infty} T / d_{\mathrm{o}}$

\section{Literature Cited}

1. Takeuchi M, Isshiki N, Ishibashi Y. Heat transfer on cylinder covered with close-fitting fabrics (Part I, wind penetration through fabrics). Bull JSME. 1982;25:1406-1411.
2. Ghaddar N, Ghali K, Jreije B. Ventilation of wind permeable clothed cylinder subject to periodic swinging motion:modeling and experimentations. J Heat Transf Trans ASME. 2008;130:091702.

3. Barry J, Hill R, Brasser P, Sobera M, Kleijn C, Gibson P. Computational fluid dynamics modeling of fabric systems for intelligent garment design. MRS Bull. 2003;28:568-573.

4. Budaraju S, Stewart W, Porter W. Mixed convection heat and moisture transfer from a horizontal furry cylinder in a transverse wind. Int J Heat Mass Transf. 1997;40:2273-2281.

5. Zhu F, Zhang W, Song G. Heat transfer in a cylinder sheathed by flame-resistant fabrics exposed to convective and radiant heat flux. Fire Saf J. 2008;43:401-409.

6. Geers L. Developments in modeling NBC protective clothing. Technical Report. PML2004-A122. Rijswijk, The Netherlands: TNOPML, 2004.

7. Fonseca G, Breckenridge J. Wind penetration through fabric systems. Text Res J. 1965;35:95-103.

8. Kind R, Jenkins J, Seddigh F. Experimental investigation of heat transfer through wind-permeable clothing. Cold Reg Sci Technol. 1991;20:39-49.

9. Kind R, Jenkins J, Broughton C. Measurements and predictions of wind-induced heat transfer through permeable cold-weather clothing. Cold Reg Sci Technol. 1995;23:305-316.

10. Bhattacharyya S, Singh A. Augmentation of heat transfer from a solid cylinder wrapped with a porous layer. Int J Heat Mass Transf. 2009;52:1991-2001

11. Sobera M, Kleijn C, Brasser P, van den Akker H. Convective heat and mass transfer to a cylinder sheathed by a porous layer. AIChE J. 2003;49:3018-3028.

12. Linders M, van der Wijst M, van Bokhoven J, Kapteijn F, Moulijn J. Design of an industrial adsorption process witch activated carbon for removal of hexafluoropropylene from wet air. Ind Eng Chem Res. 2001;40:3171-3180.

13. Brasser P. Modeling the chemical protective performance of NBC clothing material. J Occup Environ Hyg. 2004;1:620-628.

14. Brasser P, Kaaijk J. The effect of air permeability on the chemical protective performance of NBC suits. Symposium of the NATO RTO Human Factors and Medicine Panel (HFM). Brussels: NATO Research and Technology Organization, 2002.

15. Brasser P, van Houwelingen T. Theoretical and experimental study of vapor deposition onto a dressed body part. AIChE J. 2008;54:844-849.

16. Brasser P, Sobera M. Optimizing balancing protection and physiological burden of CBRN-protective clothing. In: Sparks E, editor. Advances in Military Textiles and Personal Equipment, Chapter 11. Woodhead Publishing, 2012. pp. 238-259.

17. Brasser P. Optimizing the protection against the physiological burden of CBRN-clothing. Int J Occup Saf Ergon. 2010;17:153-168.

18. Brasser P. Modeling the relation between comfort protection of CBRN-suits. In: Acosta J, Camacho A, editors. Porous Media: Heat and Mass Transfer, Transport and Mechanics. New York: Nova Science Publishers, 2009, Chapter 9. pp. 187-220.

19. Ambesi D, Kleijn C. Laminar-forced convection mass transfer to ordered and disordered single arrays of spheres. AIChE J. 2013;59: 1400-1408.

20. FLUENT Incorporated. FLUENT 6.3 Tutorial Guide 2006. Lebanon, NH: FLUENT Incorporated, 2006.

21. Gooijer H, Warmoeskerken M, Wassink J. Flow resistance of textile materials-part I: Monofilament fabrics. Text Res J. 2003;73:437-443.

22. FLUENT Incorporated. Gambit 2.2 Tutorial Guide 2004. Lebanon, NH: FLUENT Incorporated, 2004.

23. Sobera M. Hydraulic permeability of ordered and disordered singlelayer arrays of cylinders. Phys Rev E. 2006;74:paper \#036301.

24. Kee R, Coltrin M, Glarborg P. Chemically Reacting Flow - Theory and Application. Hoboken: Wiley, 2003.

25. Pope S. Turbulent Flows. Cambridge: Cambridge University Press, 2000.

26. Hanjalic K. One point closure models for buoyancy-driven turbulent flows. Annual Rev Fluid Mech. 2002;34:321-347.

27. Hanjalic K, Launder B. Modelling Turbulence in Engineering and the Environment: Second-Moment Routes to Closure. Cambridge: Cambridge University Press, 2011.

28. Hanjalic K, Kenjeres S. 'T-RANS' simulation of deterministic eddy structure in flows driven by thermal buoyancy and Lorentz force. Flow Turbul Combust. 2001;66:427-451.

29. Sobera M, Kleijn C. T-RANS Simulations of subcritical flow with heat transfer past a circular cylinder surrounded by a thin porous layer. Flow Turbul Combust. 2008;80:531-546. 
30. Hinze J. Turbulence. New York: McGraw-Hill, 1975.

31. Casey M, Wintergerste T. Quality and Trust in Industrial CFD. Wintherthur, Switzerland: ERCOFTAC-Sulzer Innotec, 2000.

32. Kuijlaars K, Kleijn C, van den Akker H. Multi-component diffusion phenomena in multiple-water chemical vapor deposition reactors. Chem Eng J Biochem Eng J. 1995;57:127-136.

33. Leonard BP, Mokhtari S. ULTRA-SHARP nonoscillatory convection schemes for high-speed steady multidimensional flow. Technical Report. NASA TM 1-2568 (ICOMP-90-12). NASA Lewis Research Center, 1990.

34. Norberg C. Fluctuating lift on a circular cylinder: Review and new measurements. J Fluids Struct. 2003;17:57-96.
35. Labraga L, Berkah T. Mass transfer from a rotating cylinder with and without crossflow. Int J Heat Mass Transf. 2004;47:2493-2499.

36. Petrie A, Simpson H. An experimental study of the sensitivity to freestream turbulence of heat transfer in wakes of cylinders in crossflow. Int J Heat Mass Transf. 1972;15:1497-1513.

37. Scholten J, Murray D. Unsteady heat transfer and velocity of a cylinder in cross flow-I. Low freestream turbulence. Int J Heat Mass Transf. 1998;41:1139-1148.

38. Sobera M, Kleijn C, van den Akker H. Subcritical flow past a circular cylinder surrounded by a porous layer. Phys Fluids. 2006;18:038106.

Manuscript received Jan. 16, 2013, revision received Jun. 17, 2013, and final revision received Sept. 29, 2013. 